\title{
Gender Differences in Distress: The Mediating Influence of Life Stressors and Psychological Resources
}

\author{
Abu Sadat Nurullah \\ Department of Sociology, University of Alberta \\ 5 - 21 Tory Building, Edmonton, Alberta, Canada T6G 2H4
}

Tel: 1-780-716-1305 E-mail: nurullah@ualberta.ca

\begin{abstract}
Using the data from Canadian National Population Health Survey (NPHS), Cycle 1, this research explore gender differences in distress and the mediating influence of life stressors (i.e., chronic stress, work stress, negative life events, and childhood and adulthood trauma), and psychological resources (self-esteem, mastery, and sense of coherence). The findings from $t$-test analyses indicated that females reported more psychological distress, chronic stress, work stress, negative life events, and childhood and adulthood trauma, compared to males. However, in terms of psychological resources, male participants reported having more self-esteem, mastery, and sense of coherence. Results from regression analyses showed that life stressors and psychological resources are mediating factors influencing gender differences in distress, controlling for other socio-demographic and psychological variables.
\end{abstract}

Keywords: Gender, Psychological distress, Life stressors, Psychological resources

\section{Introduction}

Studies on psychological distress have consistently reported gender differences: women experience more distress than men (Almeida \& Kessler, 1998; Gove \& Tudor, 1973; McDonough \& Strohschein, 2003; Mirowsky \& Ross, 1995; Mirowsky \& Ross, 1989). Researchers have explored the gender-distress relationship from cognitive-behavioral and gender role perspectives. According to the gender role perspective, women are more distressed than men because women's roles expose them to more stressors (Gove \& Tudor, 1973; McDonough \& Walters, 2001; Mirowsky \& Ross, 1989). Research from this perspective has demonstrated how differential emotional reactivity and exposure to stressors contribute to gender differences in psychological distress.

Scholars have interpreted women's distress in terms of their greater exposure to the stressors and other emotional factors. Almeida and Kessler (1998) examined men's and women's experience of daily stressors and psychological distress in a U.S. sample of 166 married couples, and concluded that the significant gender differences diminished when respondents' daily stressors were controlled for. Research on the impact of stress on mental health has focused on the role of chronic stressors (i.e., the ongoing and difficult conditions of daily life), and found that exposure to social life stress, financial stress, relationship stress, child (i.e., parenting-related) stress, environmental stress, family health stress, and job strain are positively linked to psychological distress (Denton, Prus, \& Walters, 2004).

The present research extends the study of Almeida and Kessler (1998) by including three psychological resources (self-esteem, mastery, and sense of coherence) and four life stressors (chronic stress, work stress, negative life events, and childhood and adulthood trauma). Unlike Almeida and Kessler (1998), who explored psychological distress among husbands and wives in a small U.S. sample, this study examines distress in a large national representative sample of Canadian individuals.

\section{Review of Literature}

\subsection{Empirical Studies}

Since women report greater distress than men, Mirowsky and Ross (1995) addressed the question whether women genuinely experience greater distress. Their analysis of the data from a 1990 U.S. sample of 1,282 women and 749 men showed that on an overall basis, women experienced distress about 30 percent more often than men. In addition, they found that men kept emotions to themselves more than women, and that women expressed emotions more freely than men, which may appear more distressed. Taking into consideration of the notion that drug abuse and heavy drinking might minimize male distress, their study concludes that women 
genuinely suffer more distress than men.

Previous studies reported that individuals exposed to stressful life events are at greater risk of psychological distress and psychiatric disorders (Kessler, Price \& Wortman, 1985). In their study, Almeida and Kessler (1998) found that women experienced a higher prevalence of high distress days and a lower prevalence of distress-free days than men. Research on chronic stress implied that gender differences in mental health occurred as a result of differential exposure and vulnerability to the stressors (McDonough \& Strohschein, 2003).

Denton, Prus, and Walters (2004) asserted that psychosocial determinants have both direct and mediating effects on mental health. McDonough (2000) also found that high self-esteem and mastery affected the outcomes of job stress on distress. Low self-esteem has been linked to higher levels of depression (Rosenberg, Schooler \& Schoenbach (1989). Antonovsky (1987: 140) stated that the feature of an individual with strong sense of coherence is the capacity, in confronting a stressful life state, to opt for the most suitable strategy from among the variety of prospective available resources.

\subsection{Theories}

Gender role theory emphasizes the exposure to the different types of stressors that men and women face in their daily life (Almeida \& Kessler, 1998). According to this theory, women have higher levels of distress than men because women's roles are more distress-inducing (Gove \& Tudor, 1973). Women's gender roles tend to be more nurturing, whereas men's roles are more instrumental (Gove \& Tudor, 1973). Thus, women typically are in social roles that entail them to offer more support to others, to be more considerate, and to widen their concern to a wider range of people in ways that increase stressors, which ultimately lead to distress (Gove \& Tudor, 1973; Kessler, McLeod, \& Wethington, 1985). The theory also emphasizes that gender-based health inequalities are largely the result of the differing social locations of men and women, their differing lifestyle behaviors and the differing number and levels of chronic stressors and life events experienced by men and women (Denton, Prus, \& Walters, 2004). Nonetheless, gender differences in health are only minimally reduced by controlling for exposure to structural, behavioral and psychosocial forces; significant gender differences remain (Denton, Prus, $\&$ Walters, 2004).

Research has found that chronic stressors and psychological resources play an important role in determining health, but their effects are generally stronger for women than men (Denton, Prus, \& Walters, 2004). Research also provided support for gender-distress relationship that it is mediated by role-related stressors (Almeida \& Kessler, 1998). Conger, Lorenz, Elder, Simons, and Ge (1993) showed that women are more distressed over events in the family and that men are more affected by work events.

Two alternative theories assert that women simply express themselves differently than men, thus creating a false impression of greater distress (Nolen-Hoeksema, 1987). According to the response-bias perspective, women express all emotions more freely than men. Thus, when women and men are questioned about depression and anxiety the women report it more frequently. On the other hand, the gendered-response theory says women respond to the ubiquitous stress of life with somewhat different emotions than men: men and women may experience similar levels of frustration, but men get angry at others and women get upset with themselves (Mirowsky \& Ross, 1995). If surveys ask more questions about types of distress typical of women than about those typical of men, then women may falsely appear to be more distressed.

Using the data from Canadian National Population Health Survey (NPHS) 1994-1995 (i.e., Cycle 1), this research has explored gender differences in distress and the mediating influence of life stressors (i.e., chronic stress, work stress, negative life events, and childhood and adulthood trauma), and psychological resources (self-esteem, mastery, and sense of coherence). The following two hypotheses have been tested in the study:

$\mathrm{H}_{1}$ : Women are more likely than men to experience psychological distress.

$\mathrm{H}_{2}$ : Gender differences in distress are mediated by life stressors and psychological resources.

\section{Methods}

\subsection{Data and Sample}

Data from the Canadian National Population Health Survey (NPHS) were used for this analysis. Based on a multi-stage stratified probability sampling design developed by Statistics Canada, the NPHS collects information related to the health status, use of health services, risk factors and demographic and socio-economic status of the Canadian population. In the first stage, homogeneous strata were formed and independent samples of clusters were drawn from each stratum. In the second stage, dwelling lists were prepared for each cluster and dwellings, or households, were selected from the lists. The target population of the NPHS included household residents in 
all provinces, with the principal exclusion of populations on Indian Reserves, Canadian Forces Bases, and some remote areas in Quebec and Ontario. In each household, information was collected from all household members; and one person, aged 12 years and over, from each household was randomly selected for a more in-depth interview. The NPHS questions were designed for Computer Assisted Interviewing (CAI), where questions could be customized to the respondent, based on data collected at that time.

In total, 20,725 household participated in the survey, and 17,626 people aged 12 years and older were interviewed. The household response rate at the Canada level for the NPHS was $88.7 \%$, and this rate varied from $85.2 \%$ in Ontario to $93.2 \%$ in Alberta. The selected person response rate for the NPHS was $96.1 \%$ at the Canada level, and ranged from $94.7 \%$ in Nova Scotia to $97.6 \%$ in Saskatchewan. Sample weights were applied to the data to account for unequal probabilities of selection of participants, non-response (person and household level), and the age and gender distributions of the Canadian population. The current study focused on a cross sectional sample of adults between the ages of 20 and 64 who were currently working. This restriction reduced the final sample to 9,869. Finally, due to elimination of the missing cases in the process of nesting the models, this study presents the findings based on a final sample size of 7,766 (male $=3,951$, female $=3,815$ ).

The minimum age of twenty was selected because the present study examines distress in only adult population. Since one of the stressors (i.e., work stress) requires assessing the currently working population, the maximum age of 64 was chosen, as 65 is the traditional retirement age. While the NPHS produces data for both cross-sectional and longitudinal purposes, this study is based on the 1994-1995 (i.e., Cycle 1) cross-sectional component of the NPHS because it is the only NPHS survey to date that included complete information on psychosocial resources, critical life events and chronic stressors.

\subsection{Measures}

\subsubsection{Dependent variable}

Distress was the outcome variable in this study. Distress, which is defined as an unpleasant subjective state (Ross \& Van Willigen, 1997), is based on a subset of items from the Composite International Diagnostic Interview/CIDI. Distress is a summative index derived from six items. Specifically, respondents were asked how often, during the month prior to the interview, they felt: (1) so sad that nothing could cheer you up; (2) nervous; (3) restless/fidgety; (4) hopeless; (5) worthless; and (6) everything was an effort. Responses to each item ranged from none of the time (coded as 0 ) to all of the time (4). Hence, composite scores ranged from 0 to 24 , with higher scores indicating more distress.

\subsubsection{Independent Variables}

Chronic Stress. A modified, abbreviated version of Wheaton's (1991, cited in Turner, Wheaton and Lloyd 1995) chronic stress inventory was used to measures the personal, family health, environment, child, financial and relationship stressors. In each case, a value of 0 corresponds to no reported stress. Like Turner, Wheaton, and Lloyd's (1995) study, the six dimensional items were used to form an index in this study. The index (ranging from 0 to 14) is adjusted to reflect the applicability of the items to all respondents.

Work Stress. The 12-item index, based on a larger pool of items from Karasek (1985), reflects respondents' perceptions about various dimensions of their work including job security, social support, monotony, physical effort required and extent of participation in decision-making. Sample of items include, "Your job requires that you do things over and over", "Your job security is good", and "Your job requires a lot of physical effort". Respondents' answers were based on a 5 point scale, ranging from strongly agree $=0$ to strongly disagree $=4$. The composite scores ranged from 0 to 48, with higher scores indicating greater work stress.

Recent Life Events index is based on responses to 10 events that occurred to the respondent or someone close to the respondent in the last 12 months. The events include physical abuse, unwanted pregnancy, abortion or miscarriage, major financial difficulties, and serious problems at work or school. Additionally, parents were asked about children moving back in and those living with a partner were asked about the relationship. The index (ranging from 0 to 8 ) is adjusted to reflect the applicability of the items to all respondents.

Childhood and Adult Trauma. This index measures the number of traumatic events respondents have been exposed to during their childhood, adolescence or adulthood. Events included are parental divorce, a lengthy hospital stay, prolonged parental unemployment, frequent parental alcohol or drug use. The composite scores ranged from 0 to 7 , with higher score indicating more traumatic events.

Mastery. The seven-item index which measures sense of mastery is based on the work of Pearlin and Schooler (1978). It measures the extent to which individuals believe that their life-chances are under their control. Sample of items include, "There is little you can do to change many of the important things in your life", and "You often 
feel helpless in dealing with problems of life". Respondents' answers were based on a 5 point scale, ranging from strongly agree $=0$ to strongly disagree $=4$. The composite scores ranged from 0 to 28 , with higher scores indicating superior mastery.

Self-esteem. The self-esteem index reflects the amount of positive feelings an individual holds about himself/herself. Scores on the index are based on the six items factored into one dimension in the factor analysis done by Pearlin and Schooler (1978). Sample of items include, "You feel that you have a number of good qualities", and "You take a positive attitude toward yourself". Respondents' answers were based on a 5 point scale, ranging from strongly agree $=0$ to strongly disagree $=4$. The composite scores ranged from 0 to 24 , with higher scores indicating greater self-esteem.

Sense of Coherence. The 13-item version of the sense of coherence scale developed by Antonovsky (1987) was used in the NPHS. It denotes the extent to which individuals perceive events as comprehensible, manageable and meaningful. Sample of items include, "How often do you have the feeling that you don't really care about what goes on around you?", and "How often do you have the feeling you're being treated unfairly?" The composite scores ranged from 7 to 91 , with higher score indicating a stronger sense of coherence.

Gender. Gender is a dummy variable coded 1 for males and 0 for females.

Control Variables. Age is a categorical variable and it has been recoded to the category midpoint and added as a continuous variable to the analysis. Those 20 years of age and older are the focus of this analysis. The sample was comprised of nine categories ranging from 20-24 to 60-64 years. Marital status consists of dummy variables for single (yes $=1$; no $=0$ ) and married (yes $=1$; no $=0$ ). Divorced or widowed served as the reference group. Education is years of formal schooling constructed by assigning a "years" value to each of 12 categories ranging from no schooling to medical degree or doctorate. Education consists of three dummy variables indicating the highest level of formal schooling attained: less than high school (yes $=1$; no $=0)$; completed high school (yes $=$ 1 ; no $=0$ ); and some post-secondary school (yes $=1$; no $=0$ ). Those with completed post-secondary credentials (i.e., university degree or college diploma) served as the reference group. Income is based on the respondent's report of household income for the past twelve months, adjusted for family size and expenditures on basic needs. Dummy variables were created for four levels of income: lowest income (yes $=1$; no $=0$ ); low middle income $($ yes $=1$; no $=0)$, middle income $($ yes $=1$; no $=0)$ and upper middle income (yes $=1$; no $=0$ ). Highest income households were the reference group.

\subsection{Analytic Procedure}

All analyses were conducted in SAS version 9.1. The analysis proceeded in three stages. First, chi-square and $t$-tests were conducted to assess whether there are gender differences in proportions for categorical variables, and mean scores across continuous variables. Second, bivariate regression analyses were conducted to determine the significance of the variables for inclusion in the final models. Third, ordinary least squares multiple regressions were employed to determine whether there is a mediating influence of life stressors and psychological resources factors on distress. In the end, age, the indexes of trauma, self-esteem, mastery, and coherence were treated as continuous variables in the analyses. All other independent variables were treated as categorical data, and therefore entered in the analysis as sets of dummy variables; the reference category for each set of dummy variables was shown in the tables. Because of the large number of cases in the sample, coefficients were interpreted as statistically significant if $p<.01$.

\section{Results}

Table 1 presents the weighted mean comparisons for all variables. The mean age for females was 37.94 , and the mean age for males was 38.96. Statistically significant gender differences existed in less than high school and some post-secondary level of education among the participants. More males were married compared to females, while more females were in the divorced/widowed category. However, there were no income differences between males and females.

The findings from $t$-test analyses indicated that females reported more psychological distress compared to males. Female participants were also more likely to report chronic stress, work stress, negative life events, and childhood and adulthood trauma compared to male participants. However, in terms of psychological resources, male participants reported having more self-esteem, mastery, and sense of coherence.

A central objective of this study was to examine gender differences in psychological distress. The mean differences presented in Table 1 suggest that gender differences do exist in terms of distress among the participants. Similarly, a bivariate regression analysis (not reported in this article) suggested that gender predicted psychological distress. To further examine the effect of control variables, life stressors, and 
psychological resources in gender differences on distress, three ordinary least squares regression analyses were conducted.

Table 2 shows multiple regression models for control variables, life stressors, and psychological resources outcomes. Model 1 indicates that gender significantly predicted distress. Model 2 illustrates that controlling for age, education, marital status, and income, gender still remained as a strong predictor of distress. Model 3 illustrates that gender, age, education, marital status, income, chronic stress, work stress, life events, childhood and adulthood trauma, self-esteem, mastery, and sense of coherence were all significant predictors of distress. However, in model 3, when controls, life stressors, and psychological resources variables were added, the coefficients for gender decreased somewhat, a $(.41-.18) / .41=56 \%$ reduction in distress level, but it remained significant. Adjusting for life stressors and psychological resources, only age and completed high school level of education of the control variables were significant. The model shows that life stressors and psychological resources are mediating factors influencing gender differences in distress. The final model accounted for approximately $40 \%$ of the variances in distress.

On average, men reported significantly lower levels of distress than women, adjusting for other terms in the model. The relationship between age and distress was non-linear. Increasing age was generally associated with a decline in distress with age-related declines in distress levels. All other life stressors and psychological resources variables strongly predicted distress.

\section{Discussion}

The purpose of this study was to examine gender differences in distress as well as the mediating role of four life stressors and three psychological resources. The findings supported hypothesis 1 , and suggested that females reported more psychological distress than males. The results supported and confirmed previous research findings (Almeida \& Kessler, 1998; Astbury, 2006; Gove \& Tudor, 1973; McDonough \& Walters, 2001; McDonough \& Strohschein, 2003; Mirowsky \& Ross, 1995; Mirowsky \& Ross, 1989). Astbury (2006: 380) noted that gender can explain the differences between males' and females' experience and susceptibility to specific risks to mental health, and that gender is related to the differential power of men and women to be able to respond and to cope with mental health risks. In addition, depression is one of the most recurrently encountered health problems for women which accounts for more than 1 in 10 years of life lived with disability (Astbury, 2006: 380-381). Kuehner (2003) reported that women have been more than twice as likely to be diagnosed with depression as have men across many socio-economic groups, racial groups, and cultures.

The question arises as to why women are more prone to psychological distress compared to men. Existing literature indicates various explanations for this phenomenon. Some scholars suggested that women find themselves in stressful circumstances more often than men (e.g., Almeida \& Kessler, 1998; McDonough \& Walters, 2001). Other researchers have suggested that it is possible that women assess threatening events as more stressful than men do (Ptacek, Smith, \& Dodge, 1994). Klonoff, Landrine, and Campbell (2000) suggested that women experience gender-specific stressors such as gender violence and sexist discrimination, which are associated with women's physical and psychiatric events. Furthermore, Turner, Wheaton, and Lloyd (1995) indicated that women are more affected by the stress of the people around them, as they tend to be more emotionally involved than men in social and family networks.

The findings also supported hypothesis 2: life stressors and psychological resources mediated the relations between gender and distress. This supported findings from a previous study conducted by Almeida and Kessler (1998). The final model significantly improves our understanding of gender differences in distress. Previous research indicated that the relationship between stress and health is influenced by a variety of moderator variables (Matud, 2004). The study by Matud (2004) found that women reported more stress than men when taking into account the effects of stress exposure, the enduring nature of some role-related stressors, and other long-term chronic and daily stressors. Furthermore, research has shown that men make more frequent use of instrumental coping, while women are more likely to use emotion-focused coping (Ptacek, Smith, \& Dodge, 1994). However, studies indicated that long-term stressors may reduce physical or social resources for coping with new stressors, thus making individuals more vulnerable to the harmful health effects of severe stressors (Grzywacz, Almeida, Neupert, \& Ettner, 2004).

Finally, in this research, increasing age was related to a decline in distress with age-related declines in distress levels. However, the issue of whether depression increases or decreases with age remains unclear (Riedel-Heller, Busse, \& Angermeyer, 2006). 


\subsection{Limitations}

The NPHS indeed has a longitudinal component that can be used to explore causality, but many of the social (namely psychosocial) indicators that are central to the analysis are available in the first wave (1994-1995) of the NPHS only. I do plan to conduct a longitudinal analysis as the NPHS data file matures, especially if psychosocial determinants are included in future surveys. In the end, the 1994-1995 NPHS is the only representative, large-scale/national dataset to consider the full complement of structural, behavioral and psychosocial determinants of health, and is the best available Canadian data for this particular study.

The final data is heteroscadastic and the residuals do not show a normal distribution, and therefore violates the standard assumption for least squares regression, which might limit the generalizability of the model.

\subsection{Future Direction}

As noted, the causal pathways are complex, and I have not tried to sort them out in this analysis. Now that we have established the importance of considering all the three categories as determinants of health, more detailed structural equation modeling of the direct and indirect pathways may indeed be warranted. Longitudinal data will also facilitate the exploration of the causal relationship between social forces and health. I plan to conduct such analyses when the appropriate data are available.

\section{References}

Almeida, D. M., \& Kessler, R. C. (1998). Everyday stressors and gender differences in daily distress. Journal of Personality and Social Psychology, 75(3), 670-680.

Antonovsky, A. (1987). Unravelling the mystery of health: How people manage stress and stay well. San Francisco: Jossey-Bass.

Astbury, J. (2006). Women's mental health: From hysteria to human rights. In S. E. Romans \& M. V. Seeman (Eds.), Women's mental health: A life-cycle approach (pp. 377-392). Philadelphia: Lippincott Williams \& Wilkins.

Conger, R. W., Lorenz, E. O., Elder, G. H., Simons, R. L., \& Ge, X. (1993). Husband and wife differences in response to undesirable life events. Journal of Health and Social Behavior, 34(1), 71-88.

Denton, M., Prus, S., \& Walters, V. (2004). Gender differences in health: A Canadian study of the psychosocial, structural and behavioural determinants of health. Social Science \& Medicine, 58(12), 2585-2600.

Gove, W. R., \& Tudor, J. E. (1973). Adult sex roles and mental illness. American Journal of Sociology, 78(4), 812-835.

Grzywacz, J. G., Almeida, D. M., Neupert, S. D., \& Ettner, S. L. (2004). Socioeconomic status and health: A micro-level analysis of exposure and vulnerability to daily stressors. Journal of Health and Social Behavior, 45(1), 1-16.

Karasek, R. (1985). Job content questionnaire. Los Angeles, CA: Department of Industrial and Systems Engineering, University of Southern California.

Kessler, R. C., Andrews, G., Colpe, L. J., Hiripi, E., Mroczek, D. K., et al. (2002). Short screening scales to monitor population prevalences and trends in non-specific psychological distress. Psychological Medicine, 32(6), 959-976.

Kessler, R. C., McLeod, J. D., \& Wethington, E. (1985). The costs of caring: A perspective on sex differences in psychological distress. In I. G. Sarason \& B. R. Sarason (Eds.), Social support: Theory, research, and applications (pp. 491-507). Dordrecht, the Netherlands: Martinus Nijhof.

Kessler, R. C., Price, R. H., \& Wortman, C. B. (1985). Social factors in psychopathology: stress, social support and coping processes. Annual Review of Psychology, 36, 531-572.

Kessler, R., Foster, C., Webster, P.S. \& House, J. S. (1992). The relationship between age and depressive symptoms in two national surveys. Psychology and Aging, 7(1), 119-126.

Klonoff, E. A., Landrine, H., \& Campbell, R. (2000). Sexist discrimination may account for well-known gender differences in psychiatric symptoms. Psychology of Women Quarterly, 24(1), 93-99.

Kuehner, C. (2003). Gender differences in unipolar depression: An update of epidemiological findings and possible explanations. Acta Psychiatrica Scandinavia, 108, 163-174.

Matud, M. P. (2004). Gender differences in stress and coping styles. Personality and Individual Differences, 37(7), 1401-1415. 
McDonough, P. (2000). Job insecurity and health. International Journal of Health Services, 30(3), 453-476.

McDonough, P., \& Strohschein, L. (2003). Age and the gender gap in distress. Women \& Health, 38(1), 1-20.

McDonough, P., \& Walters, W. (2001). Gender and health: reassessing patterns and explanations. Social Science \& Medicine, 52(4), 547-559.

Mirowsky, J., \& Ross, C. E. (1989). Social causes of psychological distress. New York: Aldine de Gruyter.

Mirowsky, J., \& Ross, C. E. (1995). Sex differences in distress: Real or artifact? American Sociological Review, $60(3), 449-468$.

Nolen-Hoeksema, S. (1987). Sex differences in unipolar depression: Evidence and theory. Psychological Bulletin, 101(2), 259-82.

Pearlin, L. I., \& Schooler, C. (1978). The structure of coping. Journal of Health and Social Behaviour, 19(1), $2-21$.

Ptacek, J. T., Smith, R. E., \& Dodge, K. L. (1994). Gender differences in coping with stress: When stressors and appraisal do not differ. Personality and Social Psychology Bulletin, 20(4), 421-430.

Riedel-Heller, S. G., Busse, A., Angermeyer, M. C. (2006). The state of mental health in old-age across the 'old' European Union: A systematic review. Acta Psychiatrica Scandinavica, 113(5), 388-401.

Rosenberg, M., Schooler, C., \& Schoenbach, C. (1989). Self esteem and adolescent problems: Modelling reciprocal effects. American Sociological Review, 54(6), 1004-1018.

Ross, C. E., \& Van Willigen, M. (1997). Education and the subjective quality of life. Journal of Health and Social Behaviour, 38(3), 275-297.

Turner, R.J., Wheaton, B. \& Lloyd, D. (1995). The epidemiology of social stress. American Sociological Review, $60(1), 104-125$. 
Table 1. Weighted Means and Standard Deviations, by Sex

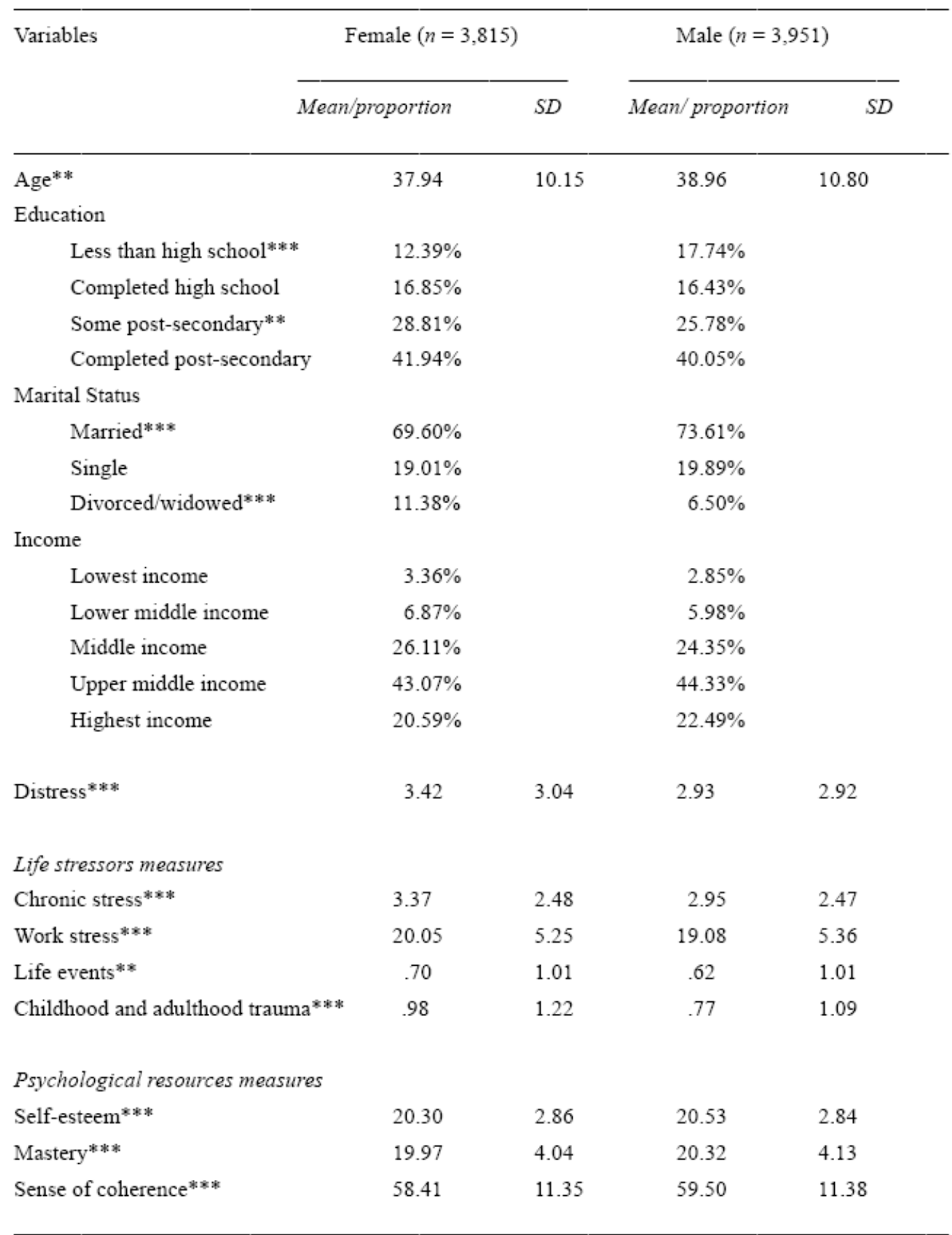

** $P<.01, * * * P<.001$ (statistical significance based on $t$-test for means and chi-square test for proportions). 
Table 2. Ordinary Least Square Regression for Control Variables, Life Stressors, and Psychological Resources Outcomes

$\begin{array}{lll}\text { Model } 1 & \text { Model } 2 & \text { Model } 3 \\ \beta \text {, (s.e.) } & \beta \text {, (s.e.) } & \beta \text {, (s.e.) }\end{array}$

$($ Female $=$ reference $)$

Male

$-.49^{* * *}(.07)$

$-.41 * * *(.07)$

$-.18^{* * *}(.05)$

Age (centered)

$-.04 * * *(.004) \quad-.02 * * *(.003)$

Education

(Post-secondary $=$ reference $)$

Less than high school

Completed high school

Some post-secondary

Marital Status

Married

Single

(Divorced/widowed $=$ reference)

Income

Lowest income

$\begin{array}{ll}.18(.20) & -.17(.16) \\ .97^{* * *}(.15) & .31(.12) \\ .35^{* * *}(.10) & -.0002(.08) \\ .09(.09) & -.09(.07)\end{array}$

Lower middle income

Middle income

Upper middle income

(Highest income $=$ reference $)$

Life stressors measures

Chronic stress (centered)

$\begin{array}{ll}.31^{* *}(.10) & -.05(.08) \\ .06(.10) & -.21 * *(.08) \\ .20(.08) & -.06(.07)\end{array}$

$-.68 * * *(.12) \quad-.14(.10)$

$-.38^{* *}(.15) \quad-.12(.12)$

Chronic stress (squared)

Work stress (centered)

Work stress (squared)

Life events

Childhood and adulthood trauma

Psychological resources measures

Mastery (centered)

Mastery (squared)

Self-esteem (centered)

Self-esteem (squared)

Sense of coherence (centered)

$.16^{* * *}(.01)$

$.01^{* *}(.003)$

$-.02 * * *(.006)$

$.0006(.0006)$

$.17^{* * *}(.03)$

$.14^{* * *}(.02)$

Sense of coherence (squared)

Constant

3.42

3.59

$-.07^{* * *}(.008)$

$.005^{* * *}(.001)$

$-.04^{* * *}(.01)$

$.01 * * *(.003)$

$-.10^{* * * *}(.003)$

Adjusted $R^{2}$

.007

.046

$.0006^{* * *}(.0002)$

$F$ value

$52.02^{* * *}$

$31.95^{* * *}$

2.85

$n$

7,766

7,766

.396

$213.26^{* * *}$

7,766

Note: Estimates are weighted. $* * P<.01,{ }^{* * *} P<.001$. 